\title{
GENERALIZATION OF THE JACKSON APPROXIMATION THEOREMS IN THE SENSE OF CH. MÜNTZ
}

\author{
BY M. VON GOLITSCHEK ${ }^{1}$ \\ Communicated by Gian-Carlo Rota, October 22, 1968
}

1. Introduction. The aim of this note is the generalization of the theorems of $\mathrm{D}$. Jackson [1]-[5] for linear combinations $\sum_{i=0}^{s} a_{i} x^{p_{i}}$.

Theorem 1 (Сн. Müntz [1], [2], [4]). Let $p_{0}, p_{1}, \cdots$ be distinct real numbers such that $0 \leqq p_{0}<p_{1}<\cdots$ and $\lim _{i \rightarrow \infty} p_{i}=\infty$. The set of powers $\left\{x^{p_{0}}, x^{p_{1}}, \cdots\right\}$ is fundamental (with the uniform norm) in $C[0,1]$ if and only if $p_{0}=0$ and $\sum_{i=1}^{\infty} 1 / p_{i}=\infty$.

Considering this theorem we ask whether the error in the $^{2}$ best uniform approximation of $f$,

$$
\tilde{E}_{s}\left(f ;\left\{p_{i}\right\}\right):=\min _{a_{i}}\left(\max _{x \in[0,1]}\left|f(x)-\sum_{i=0}^{\infty} a_{i} x^{p_{i}}\right|\right),
$$

satisfies inequalities similar to those of the Jackson theorems for the error

$$
E_{n}(f):=\min _{a_{i}}\left(\max _{x \in[a, b]}\left|f(x)-\sum_{i=0}^{n} a_{i} x^{i}\right|\right)
$$

when the exponents $p_{i}$ are of the type of Theorem 1. Our problem is therefore to find a connection between the asymptotic behaviour of the error $\tilde{E}_{s}\left(f_{;}\left\{p_{i}\right\}\right)$ for $s \rightarrow \infty$, the sequence $\left\{p_{i}\right\}$, and the "smoothness" of the function $f$. We only present our main results here. The full details will be published elsewhere.

2. Jackson theorems for polynomials $\sum_{i=0}^{i} a_{i} x^{i . r}, r>0$. We consider the sequence $p_{i}=i \cdot r, i \in N \cup\{0\}$ and $r>0$, where $N$ $=\{1,2,3, \cdots\}$. Then we approximate the function $f \in C[0,1]$ by polynomials $\tilde{P}_{8}(x)=\sum_{i=0}^{i} a_{i} x^{i \cdot r}$. We first assume that $f(x)=x^{q}$ and consider $\tilde{E}_{s}\left(x^{q} ;\{i \cdot r\}\right)$.

\footnotetext{
1 The results of this note were announced by the author in lectures held on September 16, 1967 at the German Mathematical Congress, Karlsruhe, on May 25, 1968 at the Bavarian Mathematical Congress, Eichstätt, and at the Mathematical Research Institute, Oberwolfach, Black Forest, in July 1968. The author is very grateful to Doz. Dr. P. O. Runck for helpful comments and suggestions.

2 If $p_{0}=0$, then for each function $f \in C[0,1]$ the polynomial $\sum_{i=0}^{i} a_{i} x^{p_{i}}$ of best approximation is unique since the set of functions $\left\{1, x^{p_{1}}, \cdots, x^{p_{8}}\right\}$ satisfies the Haar condition on $[0,1]$.
} 
ThEOREM 2. If $q>0$ is a positive real number and $q / r \notin N$, then

$$
\tilde{E}_{s}\left(x^{q} ;\{i \cdot r\}\right) \equiv \min _{\alpha_{i}}\left(\max _{x \in\{0,1]}\left|x^{q}-\sum_{i=0}^{\dot{1}} a_{i} x^{i, r}\right|\right)=O\left(s^{-2 q / r}\right)
$$

for $s \rightarrow \infty ;$ but $s^{e+2 q / r} \cdot \tilde{E}_{s}\left(x^{q} ;\{i \cdot r\}\right)$ is unbounded for each $\epsilon>0$.

Theorem 3. If $f \in C[0,1]$ and $\omega(f ; \delta)$ denotes the modulus of continuity of $f$, then the error $\tilde{E}_{s}(f ;\{i \cdot r\})$ has the following properties:

(a) for $r \geqq 2$ : $\tilde{E}_{s}(f ;\{i \cdot r\}) \leqq C_{r} \cdot \omega\left(f ; s^{-2 / r}\right), C_{r}=C=1+\pi^{2} / 2$,

(b) for $0<r<2: \tilde{E}_{s}(f ;\{i \cdot r\}) \leqq C_{r}^{\prime} \cdot \omega\left(f ; s^{-1}\right), C_{r}^{\prime}=C \cdot(1+1 / r)$.

THEOREM 4. Let $f \in C[0,1]$ have a continuous derivative $f^{(k)}$ of order $k \geqq 0$ in $[0,1]$ and $f^{(k)} \in \operatorname{Lip} \alpha, 0<\alpha \leqq 1$. If $1 / r \notin N$, then as $s \rightarrow \infty$

(a) for $r \geqq 2: \tilde{E}_{s}(f ;\{i \cdot r\})=O\left(s^{-\min \{(k+\alpha) \cdot 2 / r, 2 / r\}}\right)$,

(b) for $0<r<2: \tilde{E}_{s}(f ;\{i \cdot r\})=O\left(s^{-\min \mid k+\alpha, 2 / r)}\right)$.

REMARKs. (i) It is possible to show by examples that the results of Theorems 3 and 4 cannot be improved; only the constants $C_{r}$ and $C_{r}^{\prime}$ might be smaller.

(ii) The order $s^{-2 / r}$ in Theorem 4 is to be expected, since for the analytic function $f(x)=x$ the property $\tilde{E}_{s}(x ;\{i \cdot r\})=O\left(s^{-2 / r}\right)$ cannot be improved (Theorem 2).

(iii) The converses of the above $\left(p_{i}=i \cdot r\right)$ Jackson-type theorems (thus Bernstein-type theorems) are also possible.

3. Jackson theorems for polynomials $\sum_{i=0, i \notin Q}^{n} a_{i} x^{i}, Q=\left\{q_{1}, \cdots, q_{M}\right\}$ $C N$. Another important special case will now be discussed. Let $Q$ $=\left\{q_{1}, \cdots, q_{M}\right\} \subset N$ be a finite set. Considering algebraic polynomials $\widetilde{P}_{n}(x)=\sum_{i=0, i \notin Q}^{n} a_{i} x^{i}$ we derive some estimates for the error

$$
\tilde{E}_{n}(f ; \bar{Q}):=\min _{a_{i}}\left(\max _{x \in[0,1]}\left|f(x)-\sum_{i=0, i \notin Q}^{n} a_{i} x^{i}\right|\right)
$$

and we are interested in the behaviour of $E_{n}(f ; \bar{Q})$ for $n \rightarrow \infty$.

THEOREM 5. If $q \in Q$, then we have for $\tilde{E}_{n}\left(x^{q} ; \bar{Q}\right)$ defined by (3)

$$
\tilde{E}_{n}\left(x^{q} ; \bar{Q}\right)=O\left(n^{-2 q}\right), \quad n \rightarrow \infty ;
$$

but $n^{2 q+\epsilon} \cdot \tilde{E}_{n}\left(x^{q} ; \bar{Q}\right)$ is unbounded for each $\epsilon>0$.

THEOREM 6. Let $1 \leqq q_{1}<\cdots<q_{M}$.

(a) If $f \in C[0,1]$, then for $n>q_{M}$

$$
\tilde{E}_{n}(f ; \bar{Q}) \leqq A_{0} \cdot \omega\left(f ; n^{-1}\right) ;
$$

where $A_{0}=A_{0}\left(q_{1}, \cdots, q_{M}\right)$, but $A_{0}$ is independent of $f$ and $n$. 
(b) If $f \in C^{k}[0,1], k \geqq 1$, then $\widetilde{E}_{n}(f ; \bar{Q})$ satisfies the following inequality for $n>\max \left\{k, q_{M}\right\}$ :

$$
\tilde{E}_{n}(f ; \bar{Q}) \leqq A_{k} \cdot n^{-k} \cdot \omega\left(f^{(k)} ; n^{-1}\right)+B_{k} \cdot n^{-2 q^{*}},
$$

where $A_{k}=A_{k}\left(q_{1}, \cdots, q_{M}\right), \quad B_{k}=B_{k}\left(q_{1}, \cdots, q_{M} ; \quad f^{\left(q_{1}\right)}(0), \cdots\right.$, $\left.f^{\left(q_{0}\right)}(0)\right), q_{i} \leqq k$, and

$$
\begin{aligned}
q^{*} & =\min Q^{*}=\min \left\{q \in Q \mid q \leqq k, f^{(q)}(0) \neq 0\right\}, \\
& =+\infty, \quad \text { if } Q^{*}=\Phi \text { is empty. }
\end{aligned}
$$

(c) If $f \in C^{k}[0,1], k \geqq 0$ and $f^{(k)} \in \operatorname{Lip} \alpha, 0<\alpha \leqq 1$, then

$$
\tilde{E}_{n}(f ; \bar{Q})=O\left(n^{-\min \left(k+\alpha, 2 q^{*}\right)}\right), \quad n \rightarrow \infty .
$$

Now we compare Theorem 6 (c) with the estimate given by the classical Jackson theorem: $E_{n}(f)=O\left(n^{-k-\alpha}\right)$, if $E_{n}(f)$ is defined by (2). As $E_{n}(f) \leqq E_{n}(f ; \bar{Q})$ and as $\tilde{E}_{n}\left(x^{a^{*}}\right)=O\left(n^{-2 q^{*}}\right)$ for the analytic function $f(x)=x^{q^{*}}$ (if $\left.q^{*}<\infty\right)$, the order $O\left(n^{-\min \left\{k+\alpha, 22^{*}\right\}}\right)$ in Theorem $6(\mathrm{c})$ is optimal and cannot be improved.

4. Jackson theorems for polynomials $\sum_{i=0}^{i_{i}} a_{i} x^{p_{i}}$. Let $\left\{p_{i}\right\}$ be an arbitrary sequence with $0=p_{0}<p_{1}<\cdots$ and $\lim _{i \rightarrow \infty} p_{i}=\infty$. Two characteristic quantities will help us to characterize the density of this sequence $\left\{p_{i}\right\}$ in comparison with the density of $\boldsymbol{N}$.

$$
\Delta:=\liminf _{n \rightarrow \infty} \frac{\sum_{0<p_{i} \leq n} 1 / p_{i}}{\sum_{i=1}^{n} 1 / i}, \quad \tilde{\Delta}:=\limsup _{n \rightarrow \infty} \frac{\sum_{0<p_{i} \leq n} 1 / p_{i}}{\sum_{i=1}^{n} 1 / i} .
$$

We can prove important results having many applications if the sequence $\left\{p_{i}\right\}$ satisfies the following three conditions:

(4) (a) $0=p_{0}<p_{1}<\cdots$

(b) There exists a number $\Lambda>0$ with $p_{i+1}-p_{i} \geqq \Lambda$ for $i=0,1$, $2, \cdots .^{3}$

(c) $\Delta>0$.

Theorem 7. Let the sequence $\left\{p_{i}\right\}$ satisfy (4), let $q$ positive and $q \notin\left\{p_{i}\right\}_{i \in N}$, and let $\tilde{E}_{i}\left(x^{q} ;\left\{p_{i}\right\}\right)$ be defined by (1). Then for each $\epsilon>0$

$$
\tilde{E}_{s}\left(x^{q} ;\left\{p_{i}\right\}\right)=O\left(p_{i}^{-2 \Delta \cdot q+c)}\right.
$$

\footnotetext{
8 The following two Theorems 7 and 8 also remain valid if one takes $p_{i} \geqq i \cdot \Lambda$, $i \in N$, instead of $p_{i+1}-p_{i} \geqq \Lambda$.
} 
but $p_{s}^{2 \bar{q}+\iota} \cdot \hat{E}_{a}\left(x^{q} ;\left\{p_{i}\right\}\right)$ is unbounded.

THEOREM 8. Let the sequence $\left\{p_{i}\right\}$ satisfy (4) as before.

I. Case. $\tilde{\Delta} \leqq \frac{1}{2}$.

(a) If $f \in C[0,1]$, then to each $\epsilon>0$ there exists a number $N$ $=N\left(\epsilon,\left\{p_{i}\right\}\right) \in N$ such that for $s \geqq N$

$$
E_{s}\left(f ;\left\{p_{i}\right\}\right) \leqq R_{0} \cdot \omega\left(f ; p_{s}^{-2 \Delta+c)} ;\right.
$$

where $R_{0}$ is a constant, independent of $s, f,\left\{p_{i}\right\}$.

(b) If $f \in C^{k}[0,1], k \geqq 1$, then $\widetilde{E}_{s}\left(f ;\left\{p_{i}\right\}\right)$ satisfies for $s \geqq N$

$$
\tilde{E}_{s}\left(f ;\left\{p_{i}\right\}\right) \leqq R_{k} \cdot w\left(f^{(k)} ; p_{s}^{-2 \Delta+e}\right) \cdot p_{s}^{-2 \Delta k+\varepsilon k}+R_{k}^{\prime} \cdot p_{s}^{-2 \Delta q^{*}+e},
$$

where $R_{k}=R_{k}\left(\epsilon,\left\{p_{i}\right\}\right), R_{k}^{\prime}=R_{k}^{\prime}\left(\epsilon,\left\{p_{i}\right\}, f^{(\nu)}(0), 1 \leqq \nu \leqq k, \nu \notin\left\{p_{i}\right\}\right.$, and

$$
\begin{aligned}
q^{*} & =\min Q^{*}=\min \left\{q \in N \mid q \leqq k, q \notin\left\{p_{i}\right\}, \quad f^{(q)}(0) \neq 0\right\}, \\
& =+\infty, \quad \text { if } Q^{*}=\Phi .
\end{aligned}
$$

In particular, if $f \in C^{k}[0,1], k \geqq 0$, and $f^{(k)} \in \operatorname{Lip} \alpha, 0<\alpha \leqq 1$, then for each $\epsilon>0$

$$
\tilde{E}_{s}\left(f ;\left\{p_{i}\right\}\right)=\dot{p}_{s}^{*} \cdot O\left(\left(p_{c}^{-2 \Delta}\right)^{\min \left\{k+\alpha, q^{*}\right)}\right) .
$$

II. Case. $\tilde{\Delta}>\frac{1}{2}$.

(a) If $f \in C[0,1]$, then to each $\epsilon>0$ there exists a number $M$ $=M\left(\epsilon,\left\{p_{i}\right\}\right) \in N$ such that for $s \geqq M$

$$
\tilde{E}_{s}\left(f ;\left\{p_{i}\right\}\right) \leqq \tilde{R}_{0} \cdot \omega\left(f ; p_{i}^{i-\Delta / \tilde{\Delta}}\right),
$$

where $\widetilde{R}_{0}=R_{0} \cdot(1+2 \tilde{\Delta})$ is independent of $f$ and $s$.

(b) If $f \in C^{k}[0,1], k \geqq 0$, and $f^{(k)} \in \operatorname{Lip} \alpha, 0<\alpha \leqq 1$, then the error $\tilde{E}_{s}\left(f ;\left\{p_{i}\right\}\right)$ satisfies for each $\epsilon>0$

$$
\tilde{E}_{s}\left(f ;\left\{p_{i}\right\}\right)=p_{i}^{i} \cdot O\left(p_{i}^{-\min \left\{(k+\alpha) \Delta / \tilde{\Delta}, 2 \Delta q^{*}\right)}\right), \quad s \rightarrow \infty .
$$

Remarks. 1. Using Theorem 7 for $f(x)=x^{q^{*}}$ (if $q^{*}<\infty$ ) the order $p_{s}^{-2 \Delta q^{*}}$ in Theorem I(b) and II(b) is to be expected.

2. It is surprising that the cases $\tilde{\Delta} \leqq \frac{1}{2}$ and $\tilde{\Delta}>\frac{1}{2}$ have to be distinguished. But if we compare with $\S 2\left(p_{i}=i \cdot r\right)$, we notice the same phenomenon: In the case $p_{i}=i \cdot r$ the quantities $\Delta$ and $\tilde{\Delta}$ are both equal to $1 / r$ and therefore the cases

$$
r \geqq 2 \text { and } \tilde{\Delta} \leqq 1 / 2, \quad 0<r<2 \text { and } \tilde{\Delta}>1 / 2
$$

correspond to another. 
3. The quantities $\Delta$ and $\widetilde{\Delta}$ in $\S 3$ have the property $\Delta=\tilde{\Delta}=1$.

4. If we apply Theorems 7 and 8 to the particular cases treated in $\$ 2$ or $\$ 3$ and compare with the results of $\$ 2$ or $\$ 3$, we notice that they

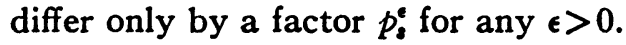

\section{REFERENCES}

1. N. I. Achieser, Vorlesungen über Approximationstheorie, Akademie-Verlag, Berlin, 1953.

2. E. W. Cheney, Introduction to approximation theory, McGraw-Hill, New York, 1966.

3. G. G. Lorentz, Approximation of functions, Holt, New York, 1966.

4. G. Meinardus, Approximation von Funktionen und ihre numerische Behandlung, Springer-Verlag, Berlin, 1964.

5. A. F. Timan, Theory of approximation of functions of a real variable, Hindustan, Delhi, 1966.

UNIVERSITY OF WÜRZBURG, GERMANY AND

Technological University of Aachen, Germany 